\title{
Comparative Evaluation of Clinical and Radiological Outcomes of Immediate and Delayed Dental Implant Rehabilitation: A Prospective Study
}

\author{
${ }^{1}$ Anil M Managutti, ${ }^{2}$ Ravish Tongya, ${ }^{3}$ Michael Prakasm, ${ }^{4}$ Supriya Manvi, ${ }^{5}$ Jigar Patel, ${ }^{6}$ Dishan Shah, ${ }^{7}$ Sunita Managutti
}

\begin{abstract}
Introduction: The original implant surgical protocol proposed by Bran Mark et al involves open flap access, stepwise osteotomy of the bony ridge, and implant placement followed by good primary closure.
\end{abstract}

Objectives: To compare the radiographic marginal bone loss, pocket depth, clinical outcome associated with immediate and delayed implants, and success rates 2 years after the placement of implants.

Materials and methods: Totally 62 dental implants were placed in 42 patients. They were randomly selected to the immediate or delayed group. Among those selected, 30 were immediate implants and 32 were delayed implants. The width and depth of marginal bone defects around the implants were measured clinically just after placement and 3 months later at the abutment surgery. Bone healing and marginal bone changes were evaluated radiographically and clinical parameters evaluated were pocket depth, local infection, altered sensation, soft tissue dehiscence, pus discharge, implant mobility, and patient's satisfaction. All the collected data were subjected to statistical analysis using Statistical Package for the Social Sciences version 17 software. Statistical analysis was done using $Z$ test.

Results: The results revealed that there was a significant difference in pocket depth and crestal bone loss in both groups. Mean pocket depth in immediate group was $3.285 \mathrm{~mm}$ and mean pocket depth in delayed group was $3.523 \mathrm{~mm}$ at 1 year. There were minimal crestal bone losses in the immediate group as compared with delayed implant group at 1-year follow-up. Also, there were reduced complications, such as local infection, altered sensation,

\footnotetext{
${ }^{1}$ Professor and Head, ${ }^{2,3,7}$ Reader, ${ }^{4}$ Professor, ${ }^{5,6}$ Postgraduate Student

1,5-7 Department of Oral and Maxillofacial Surgery, Narsinhbhai Patel Dental College \& Hospital, Sankalchand Patel University Visnagar, Gujarat, India

${ }^{2}$ Department of Prosthodontics, Index Institute of Dental Sciences, Indore, Madhya Pradesh, India

${ }^{3}$ Department of Oral and Maxillofacial Surgery, Modern Dental College \& Research Centre, Indore, Madhya Pradesh, India

${ }^{4}$ Department of Prosthodontics, KLE Society's Institute of Dental Sciences, Bengaluru, Karnataka, India

Corresponding Author: Anil M Managutti, Professor and Head Department of Oral and Maxillofacial Surgery, Narsinhbhai Patel Dental College \& Hospital, Sankalchand Patel University Visnagar, Gujarat, India, Phone: +919727756155, e-mail: dranilman12@rediffmail.com
}

soft tissue dehiscence, and pus discharge in immediate implant placement group and resulted in patient's satisfaction.

Conclusion: We conclude that the immediate dental implant placement is significantly superior over the delayed implant placement.

Keywords: Dental implants, Immediate and delayed implant placement, Osseointegration.

How to cite this article: Managutti AM, Tongya R, Prakasm M, Manvi S, Patel J, Shah D, Managutti S. Comparative Evaluation of Clinical and Radiological Outcomes of Immediate and Delayed Dental Implant Rehabilitation: A Prospective Study. Int J Oral Implantol Clin Res 2017;8(1):12-16.

\section{Source of support: Nil}

Conflict of interest: None

\section{INTRODUCTION}

Implant therapy is currently considered a successful and acceptable means to restore missing teeth. ${ }^{1}$ The best time at which to initiate implantation after dental extraction is a matter of controversy. ${ }^{2}$ Since the first report of the placement of a dental implant into a fresh extraction socket, there has been increasing interest in this technique for implant treatment. ${ }^{3}$ Traditionally, before placing dental implants, compromised teeth were removed and the extraction sockets were left to heal for between several months to 1 year. However, the great majority of patients are interested in shortening the treatment time between tooth extraction and implant placement, or even better, in having the implants inserted during the same session as the teeth are extracted.

"Immediate" implants are placed in dental sockets just after tooth extraction. "Immediate delayed" implants are those implants inserted after weeks up to about a couple of months to allow for soft tissue healing. "Delayed" implants are those placed thereafter in partially or completely healed bone. ${ }^{4}$

Advantages of immediate implants are that fewer surgical sessions and shorter treatment periods along with conservation of the crestal bone. The amount of bone loss that physiologically occurs during the remodeling phase of the extraction socket might be reduced, ${ }^{2}$ and it may not even be necessary to raise a flap and angulation of implant 
can be maintained easily. Disadvantages are risks of infections and the associated failures, if the socket is infected, lack of soft tissue closure, and flap dehiscence over the extraction site, particularly when barrier membranes have been used for guided bone regeneration. There is the necessity of raising a flap for covering the implants if a two-stage implantation procedure is preferred.

Placement of an implant into a fresh alveolus will usually result in a gap between the occlusal part of the implant and the bone walls. To ensure osseointegration of the entire implant, synthetic bone substitutes, membranes, bone grafting, osteoinductive substances, or a combination of these have been used to achieve bone formation in such defects. Autogenous bone and a variety of xenogenic graft materials have been employed in conjunction with immediate implantation, with many of them showing successful results. ${ }^{5}$

The aim of this study was to compare the radiographic marginal bone loss, pocket depth, clinical outcome associated with immediate and delayed implants, and success rates 1 year after the placement of implants in maxillary and mandibular sites, either as immediate implants or delayed implants.

\section{MATERIALS AND METHODS}

A prospective case study was made of subjects treated with immediate or delayed dental implants in the maxillary and mandibular regions between January 2008 and January 2011 and followed up to January 2012. All patients gave written informed consent prior to surgery. The study was divided in two groups - 21 patients in each group were randomly selected. The study included implants placed in 42 patients (62 implants) - 30 implants were immediate and 32 were delayed.

A protocol was prepared in which patient's detailed case history, diagnostic models and radiological and hematological investigations, implant length and diameter, type of prosthesis, buccal plate condition, and type of bone grafting used were recorded. Antibiotics and analgesics were prescribed preoperatively before 1 hour and postoperatively for 5 days. Patients with an incomplete protocol or who did not attend the follow-up examinations were excluded.

Inclusion criteria were age $\geq 18$ years, absence of relevant medical conditions contraindicating surgical interventions, requiring placement of at least one implant either immediate or delayed, and patient willing for minimum follow-up period of 12 months after implant loading. Acute periapical pathology, including vertical root fracture, endodontic failure, nonrestorable caries, or chronic periodontal disease with a hopeless prognosis were selected for placement of immediate implants after tooth extraction and at least $4 \mathrm{~mm}$ of bone apical to the extraction socket must be available.
Exclusion criteria were pregnant or lactating women, patients smoking $>10$ cigarettes / day, patients who abused alcohol or drugs, postmenopausal women with known osteoporosis, acute infection and suppuration at the fresh extraction socket, $5 \mathrm{~mm}$ or more of buccal bone loss, insufficient primary implant stability $(<25 \mathrm{Ncm})$, less than $2 \mathrm{~mm}$ of attached or keratinized gingiva, and less than 1:2 crown-implant ratio.

All surgeries were performed by the same surgeon under local anesthesia (2\% lignocaine with adrenaline1:200,000). Implant system used was the Uniti by Equinox Sales India.

\section{IMMEDIATE IMPLANT PLACEMENT}

Extractions before immediate implant placement were performed as atraumatically as possible to preserve the alveolar bone and interdental papillae. This was done with the help of peritomes. The socket was thoroughly curetted to remove any infected or inflamed tissue and remnants of the periodontal ligament. Full-thickness mucoperiosteal flaps were reflected in all cases. Drills and osteotomes were used in combination in all patients to create the implant beds. Preparation began with a round bur and a 2-mm pilot drill at $600 \mathrm{rpm}$ under irrigation with sterile water and continued with alternating osteotomes and drills until the osteotome corresponding to the implant diameter was used. Primary stability was achieved by placing the implant beyond the root apex or anchored to the socket walls. The width of the buccal bone plate and the gap between the buccal socket wall and implant were measured using a periodontal probe that was marked in millimeters.

\section{Statistical Analysis}

All the collected data were subjected to statistical analysis using Statistical Package for the Social Sciences version 17 software. Statistical analysis was done by using Z test.

\section{RESULTS}

The present study included 42 adult patients divided into two groups. The first group which is the immediate group included 14 males with mean age of 39 and 7 females with mean age of 38 years, which received 32 implants. The delayed group included 11 males with mean age of 39 and 10 females with mean age of 36 years and they received 30 implants (Table 1 ). Also, the common reason for tooth loss is caries, periodontal disease, endodontic failure, or trauma.

In our prospective study, no implants failed during follow-up. Also, no complications were observed during the periods of study. 
Table 1: Group, patient's mean age, and common etiology for tooth loss

\begin{tabular}{|c|c|c|c|c|c|}
\hline \multirow[b]{2}{*}{ Group } & \multirow[b]{2}{*}{ Male } & \multirow[b]{2}{*}{ Female } & \multicolumn{2}{|c|}{ Mean age } & \multirow[b]{2}{*}{ common etiology for tooth loss } \\
\hline & & & Male & Female & \\
\hline Immediate & 14 & 7 & 39 & 38 & Caries, PDL disease, endodontic failure \\
\hline Delayed & 11 & 10 & 39 & 36 & Caries, endodontic failure, trauma \\
\hline
\end{tabular}

PDL: Periodontal ligament

Table 2: Mean values of pocket depth and crestal bone loss in immediate group in 1-year follow-up

\begin{tabular}{llll}
\hline & 3 months $(\mathrm{mm})$ & 6 months $(\mathrm{mm})$ & 1 year $(\mathrm{mm})$ \\
\hline Pocket depth & 0.380 & 2.666 & 3.285 \\
Crestal bone loss & 0.292 & 0.590 & 0.790 \\
\hline
\end{tabular}

Table 4: $Z$ test for pocket depth

\begin{tabular}{llll}
\hline & 3 months & 6 months & 1 year \\
\hline p-value & 0.0002 & 0.017 & 0.0003 \\
Significance & Significant & Significant & Significant \\
\hline
\end{tabular}

Table 1 shows 21 patients in both groups in which immediate group comprised 14 males and 7 females, whereas delayed group comprised 11 males and 10 females randomly selected.

Tables 2 and 3 show mean pocket depth and crestal bone loss in both immediate and delayed groups at followup of 3 months, 6 months, and 1 year respectively. Table results for mean pocket depth at follow-up of 6 months and 1 year in immediate and delayed groups show similar values, but crestal bone loss at follow-up of 6 months and 1 year shows greater results in immediate group. There is very minimal crestal bone loss in immediate implant placement group as compared with the delayed implant placement group. This suggests great implant survival and stability in the immediate implant placement group.

Tables 4 and 5 present the $Z$ test for comparing pocket depth and crestal bone loss and show $p$ values. There is
Table 3: Mean values of pocket depth and crestal bone loss in delayed group in 1-year follow-up

\begin{tabular}{llll}
\hline & 3 months $(\mathrm{mm})$ & 6 months $(\mathrm{mm})$ & 1 year $(\mathrm{mm})$ \\
\hline Pocket depth & 2 & 2.785 & 3.523 \\
Crestal bone loss & 0.202 & 0.690 & 1.178 \\
\hline
\end{tabular}

Table 5: $Z$ test for crestal bone loss

\begin{tabular}{llll}
\hline & 3 months & 6 months & 1 year \\
\hline p-value & 0.0431 & 0.0141 & 0.0474 \\
Significance & Significant & Significant & Significant \\
\hline
\end{tabular}

highly significant difference in values in both pocket depth and crestal bone loss.

Graph 1 shows clinical evaluation in terms of local infection, altered sensation, soft tissue dehiscence, pus discharge, and implant stability. Altered sensation, pus discharge, and implant mobility were absent in both immediate and delayed groups, whereas local infection and soft dehiscence were minimally noted in immediate implant group as compared with delayed implant group.

Graph 2 shows patient satisfaction including esthetics. We used the patient's answers on a $100-\mathrm{mm}$ visual analog scale (VAS). On the VAS, the most negative expression corresponded to 0 and the most positive to 100 based on the following questions:

- How did you experience the period between tooth extraction and insertion of the implant crown?

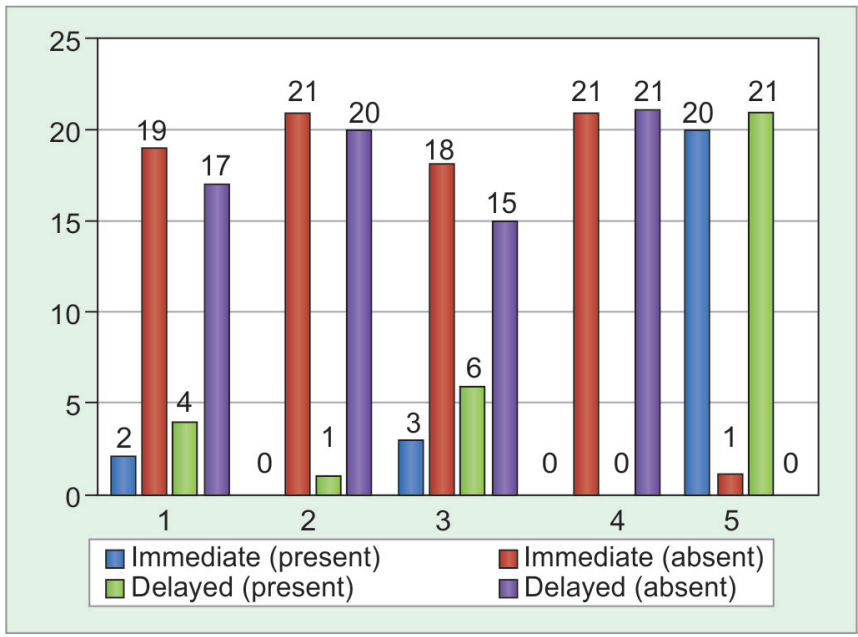

Graph 1: Clinical parameter

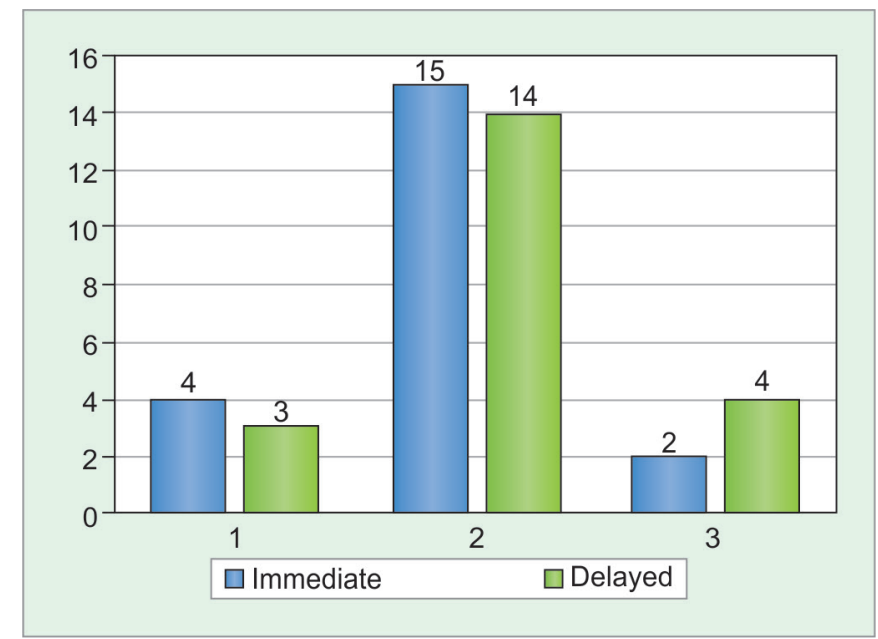

Graph 2: Patient's satisfaction 
- Are you in general satisfied with the appearance of the crown?

- How was your experience of the overall treatment?

\section{DISCUSSION}

Immediate implants have predictable results with several advantages over delayed implant placements. Over the last few years, the immediate placement of dental implant has become a subject of interest for dental professionals. Several studies concerning immediate dental implant have helped this technique to become a routine clinical procedure. $^{6}$

Over time, clinical experience has provided the criteria for immediate implant treatment success: atraumatic tooth extraction, sterilization and minimal invasive surgical approach, as well as implant primary stability. ${ }^{7}$

Quirynen et al focused their review on immediate vs delayed implant placement. Most papers contained only data on implant loss, but did not provide useful information on implant failure or hard and soft tissue changes. Their data match the results of the present review, in which most of the articles reported data on implant survival rates but not on implant success rates, according to the criteria described by Albrektsson et al. ${ }^{7}$

Depending on the degree of damage of the extraction socket and of the shape as well as the diameter of the extracted root, some portion of the implants could remain exposed and/or there might remain a residual gap between the implant and the bony wall. Since alveolar bone will remodel after tooth extraction, the degree of bone resorption is difficult to predict and could leave some portion of the implants exposed, determining a poor esthetic outcome. .,8-10 $^{\text {, }}$

According to the fourth ITI Consensus Conference (November 2009), immediate implant placement is a more difficult technique than delayed implant placement to allow initial stability and a good prosthetic position. There is also an increased risk of mucosal recession. Nonetheless, based on the esthetic index, $80 \%$ of immediate implant sites show satisfactory outcomes. The survival rates of postextraction implants are high and comparable with those of implants placed in healing sites. 7,11

A few reviews ${ }^{3-5}$ evaluating the efficacy of immediate implants have been published over the years, but so far evidence was inconclusive. It would be of great benefit to know whether the number of surgical sessions, treatment time, and patient discomfort may be reduced using immediate implants without compromising on the success of the implant therapy. ${ }^{12}$

Several reviews reported that the immediate implant treatment using autogenous bone grafts or xenografts may improve the process of bone formation between the implant and the surrounding socket walls as well as survival rates. ${ }^{13}$

With regard to the gap between the socket wall and the implant, it was reported that if the jumping distance is over $2 \mathrm{~mm}$, grafting is recommended. Smaller distances could heal spontaneously. 7,14

Based on review of the literature tackled, immediate implant placement following tooth extraction might be a viable alternative to delayed placement. However, it requires a careful case selection and a specific treatment protocol because it is a very sensitive technique and more difficult to execute than a conventional protocol. ${ }^{15}$

\section{CONCLUSION}

From the results of the present study, it can be concluded that the immediate dental implant placement is significantly superior over the delayed implant placement. Also, placement of immediate implants into extraction sockets reduces the process of alveolar bone resorption and treatment time.

However, with good case selection following detailed clinical and radiographic examinations, adequate clinical experience and judgment, this protocol, which is well received by patients, can lead to successful outcomes both esthetically and functionally.

\section{REFERENCES}

1. Horwitz J, Machtei EE. Immediate and delayed restoration of dental implants in patients with a history of periodontitis: a prospective evaluation up to 5 years. Int J Oral Maxillofac Implants 2012 Sep-Oct;27(5):1137-1143.

2. Schultes G, Gaggl A. histologic evaluation of immediate versus delayed placement of implants after tooth extraction. Oral Surg Oral Med Oral Pathol Oral Radiol Endod 2001 Jul;92(1):17-22.

3. Chen ST, Wilson TG Jr, Hämmerle CH. Immediate or early placement of implants following tooth extraction: review of biologic basis, clinical procedures, and outcomes. Int J Oral Maxillofac Implants 2004 Feb;19 (Suppl):12-25.

4. Esposito M, Grusovin MG, Polyzos IP, Felice P, Worthington HV. Interventions for replacing missing teeth: dental implants in fresh extraction sockets (immediate, immediate-delayed and delayed implants). Cochrane Database Syst Rev 2010 Sep;9:CD005968.

5. Schropp L, Kostopoulos L, Wenzel A. Bone healing following immediate versus delayed placement of titanium implants into extraction sockets: a prospective clinical study. Int J Oral Maxillofac Implants 2003 Mar-Apr;18(2):189-199.

6. Hassan KS, Kassim A, Al Ogaly AU. A comparative evaluation of immediate dental implant with autogenous versus synthetic guided bone regeneration. Oral Surg Oral Med Oral Pathol Oral Radiol Endod 2008 Nov;106(5):e8-e15.

7. Ortega-Martínez J, Pérez-Pascual T, Mareque-Bueno S, Hernández-Alfaro F, Ferrés-Padró E. Immediate implants following tooth extraction. A systematic review. Med Oral Patol Oral Cir Bucal 2012 Mar;17(2):e251-e261.

8. Ross S, Pette GA. Immediate implant placement and provisionalization using a customized anatomic temporary abutment 
(CATA) to achieve gingival marginal stability. Compendium 2013 May;34(5).

9. Yong LT. Single stage immediate implant placements in the esthetic zone. J Oral Implantol 2012 Dec;38(6):738-746.

10. Levin BP, Wilk BL. Immediate provisionalization of immediate implants in the esthetic zone: a prospective case series evaluating implant survival, esthetics, and bone maintenance. Compend Contin Educ Dent. 2013 May;34(5):352-361.

11. Romanos G, Froum S, Hery C, Cho SC, Tarnow D. Survival rate of immediately' vs delayed loaded implants: analysis of the current literature. J Oral Implantol 2010;36(4): 315-324.

12. Kaptein ML, Hoogstraten J, de Putter C, de Lange GL, Blijdorp PA. Dental implants in the atrophic maxilla: measurements of patients' satisfaction and treatment experience. Clin Oral Impl Res 1998 Oct;9(5):321-326.

13. Novaes AB Jr, Suaid F, Queiroz AC, Muglia VA, Souza SL, Palioto DB, Taba M Jr, Grisi MF. Buccal bone plate remodeling after immediate implant placement with and without synthetic bone grafting and flapless surgery: radiographic study in dogs. J Oral Implantol 2012 Dec:38(6):687-699.

14. Park JB. Implant installation with bone augmentation and transmucosal healing with demineralized human cortical bone in the maxillary anterior region: report of 3 cases. J Oral Implantol 2012 Dec;38(6):762-766.

15. Butler BL, Kinzer G. The team approach to esthetic immediate implant placement. Restorative Dentist/Periodontist Partnership 2012 Oct;33(9). 